\title{
Article \\ Existence and Uniqueness Theorem for Uncertain Wave Equation
}

\author{
Rong Gao (D)
}

School of Economics and Management, Hebei University of Technology, Tianjin 300401, China; rgao@hebut.edu.cn

\begin{abstract}
In the real world, the indeterminate phenomenon and determinate phenomenon are symmetric; however, the indeterminate phenomenon absolutely exists. Hence, the indeterminate dynamic phenomenon is studied in this paper by using uncertainty theory, where the indeterminate dynamic phenomenon is associated with the belief degree and called the uncertain dynamic phenomenon. Based on uncertainty theory, the uncertain wave equation derived by the Liu process is constructed to model the propagation of various types of wave with uncertain disturbance in nature, where the Liu process is Lipschitz-continuous and has stationary and independent increments. First important of all, only the equation has solution which can be used to clearly depict the wave propagation influenced by uncertain disturbance. Therefore, the aims of this paper is to propose and prove a theorem of existence and uniqueness with Lipschitz and linear growth conditions.
\end{abstract}

Keywords: existence and uniqueness; Liu process; uncertain wave equation; partial differential equation

\section{Introduction}

Uncertainty theory was founded by Liu [1] to model the indeterminacy associated with belief degrees. A belief degree is a chance that a possible event may happen and the chance is estimated by a human. So, uncertainty theory could be regarded as an instrument to describe uncertain phenomena in human systems. For example, Liu [2] uses uncertain variable to model indeterminate factors and established uncertain programming, which has been applied to green supply chain management [3]. After uncertainty theory was constructed, some other scholars had also done much meaningful work in many fields, such as reliability analysis [4], portfolio optimization [5,6], vehicle routing problems [7] and uncertain risk analysis [8].

An uncertain process is a collection of uncertain variables varying with space or time to model dynamic uncertain phenomena. Its investigation was pioneered by Liu [9]. Following that, Liu [10] proposed the Liu process, a special type of uncertain process, which is Lipschitz-continuous and has independent and stationary increments which are actually uncertain normal variables. On the base of the Liu process, Liu [10] initialized uncertain calculus to handle integration and differentiation of some uncertain processes.

The uncertain differential equation was established by Liu [9] which is one kind of differential equation motivated by the Liu process. After that, when satisfying linear growth and Lipschitz continuity, Chen and Liu [11] presented and verified the existence and uniqueness theorem of an uncertain differential equation. Following that, the property of stability was studied, including stability in uncertain measures [10], stability in the $p$ th moment [12] and stability in mean [13]. Additionally, it is significant for us to obtain the solution. Hence, the analytic solution was studied in [11] for a linear uncertain differential equation and two special classes of nonlinear differential equations, respectively. To obtain the numerical solution, the Yao-Chen formula was derived by Yao and Chen [14] by which we can derive a class of equivalent ordinary differential equations. Then, Gao [15] applied the Yao-Chen formula to design an algorithm and obtain the numerical solution of an 
uncertain differential equation. Recently, it has been used in high numbers of research fields, such as finance [16] and optimal control [17].

The partial differential equation has been applied widely in many fields, while disturbances are always around us in real world. So, deterministic partial differential equations are unable to model some phenomena with indeterminate disturbance. With the development of the Wiener Process, the partial differential equation found it was extended to the stochastic partial differential equation. For example, Walsh [18] investigated the stochastic heat equation for dealing with heat conduction. In addition, Cabaña [19] studied the stochastic wave equation driven by a white noise for modeling wave propagation phenomena. Then stochastic wave equation was also studied by Orsingher [20], Marcus [21] and Czapor [22].

Probability theory is employed to describe randomness in relation to frequencies, while uncertainty theory is applied to model uncertainty associated with belief degrees. So, to describe phenomena with uncertain disturbance, Yang and Yao [23] introduced uncertainty theory into the partial differential equation and the presented uncertain partial differential equation. They also studied the uncertain heat equation, a type of uncertain differential equation. In addition, Gao and Ralescu [24] established the uncertain wave equation involving the Liu process to describe wave propagation influenced by uncertain disturbance. In this paper, the existence and uniqueness theorem will be presented and proved regarding a nonlinear uncertain wave equation. Moreover, the paper will be arranged as follows. Section 2 will introduce some necessarily fundamental knowledge used in other sections. Then we show the process of proof of the existence and uniqueness theorem with some conditions and give some examples in Section 3. Ultimately, a concise conclusion is drawn in Section 4.

\section{Preliminaries}

The uncertain wave equation involving the Liu process plays a significant role in modeling wave propagation with uncertain disturbances. Consequently, some basic knowledge will be introduced firstly.

Definition 1 (Liu [9]). Suppose that $T$ is a totally ordered set and suppose that $(\Gamma, \mathcal{L}, \mathcal{M})$ is an uncertainty space. If function $X_{\tau}: T \times(\Gamma, \mathcal{L}, \mathcal{M}) \rightarrow \Re$ is measurable, that is, $\left\{X_{\tau} \in B\right\}$ is an event for each Borel set $B$ for every $t$, then $X_{\tau}$ is called an uncertain process.

If $X_{\tau+s}-X_{s}$ have a common distribution for each $\tau>0$ and $s>0$, then we say that $X_{\tau}$ is a stationary uncertain process. If for any $\tau_{1}, \tau_{2}, \cdots, \tau_{k}$ with $\tau_{0}<\tau_{1}<\cdots<\tau_{k}, X_{\tau_{0}}, X_{\tau_{1}}-$ $X_{\tau_{0}}, \cdots, X_{\tau_{k}}-X_{\tau_{k-1}}$ are independent, then we say $X_{\tau}$ has independent increments, where $\tau_{0}$ is the initial time.

Definition 2 (Liu [10]). If the uncertain process $C_{\tau}$ satisfies the following conditions

(i) Almost all of its sample paths are Lipschitz continuous and $C_{0}=0$;

(ii) $C_{\tau}$ has independent increments and is also stationary;

(iii) The expected value and variance of every increment $C_{s+\tau}-C_{s}$ are 0 and $\tau^{2}$, and uncertainty distribution of $C_{s+\tau}-C_{s}$ is

$$
\Phi(x)=\left(1+\exp \left(\frac{-\pi x}{\sqrt{3} \tau}\right)\right)^{-1}, x \in \Re,
$$

then $C_{\tau}$ is called the Liu process.

Definition 3 (Liu [10]). Assume that $C_{\tau}$ is a Liu process and assume that $X_{\tau}$ is an uncertain process. For any partition of $[a, b]$ satisfying $a=\tau_{1}<\tau_{2}<\cdots<\tau_{n+1}=b$, we write the net as follows

$$
\Delta=\max _{1 \leq i \leq n}\left|\tau_{i+1}-\tau_{i}\right| .
$$


Then the Liu integral of $X_{\tau}$ in relation to $C_{\tau}$ is

$$
\int_{a}^{b} X_{\tau} \mathrm{d} C_{\tau}=\lim _{\Delta \rightarrow 0} \sum_{i=1}^{n} X_{\tau_{i}} \cdot\left(C_{\tau_{i+1}}-C_{\tau_{i}}\right)
$$

providing that the limit almost surely exists and it is finite. Furthermore, we say $X_{\tau}$ integrable.

Definition 4 (Liu [9]). Let $C_{\tau}$ be a Liu process. Assume that functions $g$ and $h$ are continual. Then

$$
\mathrm{d} X_{\tau}=g\left(\tau, X_{\tau}\right) \mathrm{d} \tau+h\left(\tau, X_{\tau}\right) \mathrm{d} C_{\tau}
$$

is called an uncertain differential equation.

Definition 5 (Liu [25]). Suppose there is a partially ordered set $T$ and an uncertainty space $(\Gamma, \mathcal{L}, \mathcal{M})$. If the function $X_{\tau}(\gamma): T \times(\Gamma, \mathcal{L}, \mathcal{M}) \rightarrow \Re$ is measurable, that is, for any Borel set $B$ $\left\{X_{\tau} \in B\right\}$ is an event for each $\tau$, then $X_{\tau}$ is said to be an uncertain field.

\section{Existence and Uniqueness}

This section is employed to show a theorem regarding existence and uniqueness for the uncertain nonlinear wave equation [24] as follows.

$$
\left\{\begin{array}{l}
\frac{\partial^{2} X}{\partial \tau^{2}}(\tau, y)=a^{2} \frac{\partial^{2} X}{\partial y^{2}}(\tau, y)+g(\tau, y, X(\tau, y))+h(\tau, y, X(\tau, y)) \dot{C_{\tau}},(\tau, y) \in \Re^{+} \times \Re \\
X(0, y)=\psi(y), y \in \Re \\
\frac{\partial X}{\partial \tau}(0, y)=\varphi(y), y \in \Re .
\end{array}\right.
$$

where $\dot{C}_{\tau}=\mathrm{d} C_{\tau} / \mathrm{d} \tau$. Then, Equation (5) is equivalent to

$$
\begin{aligned}
X(\tau, y)= & \frac{1}{2}[\psi(y+a \tau)+\psi(y-a \tau)]+\frac{1}{2 a} \int_{y-a \tau}^{y+a \tau} \varphi(z) \mathrm{d} z+\frac{1}{2 a} \int_{0}^{\tau} \mathrm{d} t \int_{y-a(\tau-t)}^{y+a(\tau-t)} \\
& g(\tau, z, X(t, z)) \mathrm{d} z+\frac{1}{2 a} \int_{0}^{\tau} \mathrm{d} C_{t} \int_{y-a(\tau-t)}^{y+a(\tau-t)} h(t, z, X(t, z)) \mathrm{d} z .
\end{aligned}
$$

Firstly, a lemma is introduced before proving the theorem of existence and uniqueness.

Lemma 1 (Chen and Liu [11]). Let the uncertain process $X_{\tau}$ be integrable on $[a, b]$ in relation to $\tau$ and assume that $C_{\tau}$ is a Liu process. Then, we have

$$
\left|\int_{a}^{b} X_{\tau}(\gamma) \mathrm{d} C_{\tau}(\gamma)\right| \leq K_{\gamma} \int_{a}^{b}\left|X_{\tau}(\gamma)\right| \mathrm{d} \tau .
$$

Here, $K_{\gamma}$ is the Lipschitz constant of $X_{\tau}(\gamma)$.

Theorem 1. The solution of Equation (5) is unique if $\psi(y)$ and $\varphi(y)$ satisfy

$$
|\psi(y)| \leq P,|\varphi(y)| \leq Q, \forall y \in \Re
$$

where $P, Q>0, g(\tau, y, x)$ and $h(\tau, y, x)$ satisfy

$$
|g(\tau, y, x)-g(\tau, y, v)|+|h(\tau, y, x)-h(\tau, y, v)| \leq L|x-v|, \forall x, v \in \Re, \tau \geq 0
$$

and

$$
|g(\tau, y, x)|+|h(\tau, y, x)| \leq L(1+|x|), \forall x \in \Re, \tau \geq 0
$$

for some crisp number L. Furthermore, the solution of Equation (5) is sample-continuous. 
Proof. Firstly, we testify the existence through the method of successive approximation. Conveniently, $X(\tau, y)$ is simplified as $X_{\tau, y}$.

Let

$$
X_{\tau, y}^{(0)}=X_{0, y}=\psi(y),
$$

and

$$
\begin{aligned}
X_{\tau, y}^{(n)}= & \frac{1}{2}[\psi(y+a \tau)+\psi(y-a \tau)]+\frac{1}{2 a} \int_{y-a \tau}^{y+a \tau} \varphi(z) \mathrm{d} z+\frac{1}{2 a} \int_{0}^{\tau} \mathrm{d} t \int_{y-a(\tau-t)}^{y+a(\tau-t)} g\left(t, z, X_{\tau, z}^{(n-1)}\right) \mathrm{d} z \\
& +\frac{1}{2 a} \int_{0}^{\tau} \mathrm{d} C_{t} \int_{y-a(\tau-t)}^{y+a(\tau-t)} h\left(t, z, X_{\tau, z}^{(n-1)}\right) \mathrm{d} z, n=1,2, \cdots
\end{aligned}
$$

Define

$$
D_{\tau}^{(n)}(\gamma)=\max _{y \in \Re} \max _{0 \leq s \leq \tau}\left|X_{s, y}^{(n+1)}(\gamma)-X_{s, y}^{(n)}(\gamma)\right|, \forall \gamma \in \Gamma .
$$

By Equation (9) and Lemma 1, we have

$$
\begin{aligned}
& D_{\tau}^{(0)}(\gamma)=\max _{y \in \Re} \max _{0 \leq s \leq \tau} \mid \frac{1}{2}[\psi(y+a s)+\psi(y-a s)]+\frac{1}{2 a} \int_{y-a s}^{y+a s} \varphi(z) \mathrm{d} z \\
& +\frac{1}{2 a} \int_{0}^{s} \mathrm{~d} t \int_{y-a(s-t)}^{y+a(s-t)} g\left(t, z, X_{t, z}^{(0)}(\gamma) \mathrm{d} z\right. \\
& +\frac{1}{2 a} \int_{0}^{s} \mathrm{~d} C_{t}(\gamma) \int_{y-a(s-t)}^{y+a(s-t)} h\left(t, z, X_{t, z}^{(0)}(\gamma)\right) \mathrm{d} z-\psi(y) \\
& \leq 2 P+\max _{y \in \Re} \max _{0 \leq s \leq \tau}\left|\frac{1}{2 a} \int_{y-a s}^{y+a s} \varphi(z) \mathrm{d} z\right|+\max _{y \in \Re} \max _{0 \leq s \leq \tau} \mid \frac{1}{2 a} \int_{0}^{s} \mathrm{~d} t \int_{y-a(s-t)}^{y+a(s-t)} \\
& g(t, z, \psi(z)) \mathrm{d} z\left|+\max _{y \in \Re} \max _{0 \leq s \leq \tau}\right| \frac{1}{2 a} \int_{0}^{s} \mathrm{~d} C_{t}(\gamma) \int_{y-a(s-t)}^{y+a(s-t)} h(t, z, \psi(z)) \mathrm{d} z \mid \\
& \leq 2 P+\max _{y \in \Re} \max _{0 \leq s \leq \tau} \frac{1}{2 a} \int_{y-a s}^{y+a s}|\varphi(z)| \mathrm{d} z+\max _{y \in \Re} \max _{0 \leq s \leq \tau} \frac{1}{2 a} \int_{0}^{s} \mathrm{~d} t \int_{y-a(s-t)}^{y+a(s-t)} \\
& |g(t, z, \psi(z))| \mathrm{d} z+\max _{y \in \Re} \max _{0 \leq s \leq \tau} \frac{1}{2 a} \int_{0}^{s} \mathrm{~d} t \int_{y-a(s-t)}^{y+a(s-t)} K_{\gamma}|h(t, z, \psi(z))| \mathrm{d} z \\
& \leq 2 P+Q \tau+\max _{y \in \Re} \max _{0 \leq s \leq \tau} \frac{1}{2 a} \int_{0}^{s} \mathrm{~d} t \int_{y-a(s-t)}^{y+a(s-t)} L(1+|\psi(z)|)\left(1+K_{\gamma}\right) \mathrm{d} z \\
& \leq 2 P+Q \tau+\max _{y \in \Re} \int_{0}^{\tau} L(1+P)\left(1+K_{\gamma}\right)(\tau-t) \mathrm{d} t \\
& =2 P+Q \tau+\frac{L(1+P)\left(1+K_{\gamma}\right)}{2} \tau^{2} .
\end{aligned}
$$

For $n=1,2, \cdots$, we claim that

$$
D_{\tau}^{(n)}(\gamma) \leq \frac{2 L^{n}\left(1+K_{\gamma}\right)^{n} P}{(2 n) !} \tau^{2 n}+\frac{L^{n}\left(1+K_{\gamma}\right)^{n} Q}{(2 n+1) !} \tau^{2 n+1}+\frac{L^{n+1}\left(1+K_{\gamma}\right)^{n+1}(1+P)}{(2 n+2) !} \tau^{2 n+2},
$$


where $0 \leq \tau \leq T$ and $T$ is a crisp number. If $k=1$, it follows from Equations (11) and (12) and Lemma 1 that

$$
\begin{aligned}
& D_{\tau}^{(1)}(\gamma)=\max _{y \in \Re} \max _{0 \leq s \leq \tau} \mid \frac{1}{2 a} \int_{0}^{s} \mathrm{~d} t \int_{y-a(s-t)}^{y+a(s-t)}\left[g\left(t, z, X_{t, z}^{(1)}(\gamma)\right)-g\left(t, z, X_{t, z}^{(0)}(\gamma)\right)\right] \mathrm{d} z \\
& +\frac{1}{2 a} \int_{0}^{s} \mathrm{~d} C_{t}(\gamma) \int_{y-a(s-t)}^{y+a(s-t)}\left[h\left(t, z, X_{t, z}^{(1)}(\gamma)\right)-h\left(t, z, X_{t, z}^{(0)}(\gamma)\right)\right] \mathrm{d} z \mid \\
& \leq \max _{y \in \Re} \max _{0 \leq s \leq \tau}\left|\frac{1}{2 a} \int_{0}^{s} \mathrm{~d} t \int_{y-a(s-t)}^{y+a(s-t)}\left[g\left(t, z, X_{t, z}^{(1)}(\gamma)\right)-g\left(t, z, X_{t, z}^{(0)}(\gamma)\right)\right] \mathrm{d} z\right| \\
& +\max _{y \in \Re} \max _{0 \leq s \leq \tau}\left|\frac{1}{2 a} \int_{0}^{s} \mathrm{~d} C_{t}(\gamma) \int_{y-a(s-t)}^{y+a(s-t)}\left[h\left(t, z, X_{t, z}^{(1)}(\gamma)\right)-h\left(t, z, X_{t, z}^{(0)}(\gamma)\right)\right] \mathrm{d} z\right| \\
& \leq \max _{y \in \Re} \max _{0 \leq s \leq \tau} \frac{1}{2 a} \int_{0}^{s} \mathrm{~d} t \int_{y-a(s-t)}^{y+a(s-t)}\left|g\left(t, z, X_{t, z}^{(1)}(\gamma)\right)-g\left(t, z, X_{t, z}^{(0)}(\gamma)\right)\right| \mathrm{d} z \\
& +\max _{y \in \Re} \max _{0 \leq s \leq \tau} \frac{1}{2 a} \int_{0}^{s} \mathrm{~d} C_{t}(\gamma) \int_{y-a(s-t)}^{y+a(s-t)}\left|h\left(t, z, X_{t, z}^{(1)}(\gamma)\right)-h\left(t, y, X_{t, z}^{(0)}(\gamma)\right)\right| \mathrm{d} z \\
& \leq \max _{y \in \Re} \max _{0 \leq s \leq \tau} \frac{1}{2 a} \int_{0}^{s} \mathrm{~d} t \int_{y-a(s-t)}^{y+a(s-t)}\left|g\left(t, z, X_{t, z}^{(1)}(\gamma)\right)-g\left(t, z, X_{t, z}^{(0)}(\gamma)\right)\right| \mathrm{d} z \\
& +\max _{y \in \Re} \max _{0 \leq s \leq \tau} \frac{1}{2 a} \int_{0}^{s} \mathrm{~d} t K_{\gamma} \int_{y-a(s-t)}^{y+a(s-t)}\left|h\left(t, z, X_{t, z}^{(1)}(\gamma)\right)-h\left(t, z, X_{t, z}^{(0)}(\gamma)\right)\right| \mathrm{d} z .
\end{aligned}
$$

Then, by using Equation (8) and Equation (13), we have

$$
\begin{aligned}
D_{\tau}^{(1)}(\gamma) \leq & \left.\left.\max _{y \in \Re} \max _{0 \leq s \leq \tau} \frac{1}{2 a} \int_{0}^{s} \mathrm{~d} t \int_{y-a(s-t)}^{y+a(s-t)} L \mid X_{t, z}^{(1)}(\gamma)\right)-X_{t, z}^{(0)}(\gamma)\right) \mid \mathrm{d} z \\
& \left.\left.+\max _{y \in \Re} \max _{0 \leq s \leq \tau} \frac{K_{\gamma}}{2 a} \int_{0}^{s} \mathrm{~d} t \int_{y-a(s-t)}^{y+a(s-t)} L \mid X_{t, z}^{(1)}(\gamma)\right)-X_{t, z}^{(0)}(\gamma)\right) \mid \mathrm{d} z \\
= & \left.\left.\frac{1+K_{\gamma}}{2 a} L \max _{y \in \Re} \max _{0 \leq s \leq \tau} \int_{0}^{s} \mathrm{~d} t \int_{y-a(s-t)}^{y+a(s-t)} \mid X_{t, z}^{(1)}(\gamma)\right)-X_{t, z}^{(0)}(\gamma)\right) \mid \mathrm{d} z \\
\leq & \frac{1+K_{\gamma}}{2 a} L \max _{y \in \Re} \max _{0 \leq s \leq \tau} \int_{0}^{s} \mathrm{~d} t \int_{y-a(s-t)}^{y+a(s-t)}\left[2 P+Q t+\frac{(1+P)\left(1+K_{\gamma}\right)}{2} L t^{2}\right] \mathrm{d} z \\
\leq & \frac{1+K_{\gamma}}{2 a} L \int_{0}^{\tau} 2 a(\tau-t)\left[2 P+Q t+\frac{\tau^{2}}{2} L(1+P)\left(1+K_{\gamma}\right)\right] \mathrm{d} t \\
= & L\left(1+K_{\gamma}\right)\left[P \tau^{2}+\frac{Q}{6} \tau^{3}+\frac{L\left(1+K_{\gamma}\right)(1+P)}{24} \tau^{4}\right] .
\end{aligned}
$$

Equation (14) holds for $k=1$ via Equation (16). If we think that Equation (14) holds for $k=n-1$, then we will prove that it also holds for $k=n$ according to the induction method. Through Equations (11) and (12) and Lemma 1, we derive 


$$
\begin{aligned}
D_{\tau}^{(n)}(\gamma)= & \max _{y \in \Re} \max _{0 \leq s \leq \tau} \mid \frac{1}{2 a} \int_{0}^{s} \mathrm{~d} t \int_{y-a(s-t)}^{y+a(s-t)}\left[g\left(t, z, X_{t, z}^{(n)}(\gamma)\right)-g\left(t, z, X_{t, z}^{(n-1)}(\gamma)\right)\right] \mathrm{d} z \\
& +\frac{1}{2 a} \int_{0}^{s} \mathrm{~d} C_{t}(\gamma) \int_{y-a(s-t)}^{y+a(s-t)}\left[h\left(t, z, X_{t, z}^{(n)}(\gamma)\right)-h\left(t, z, X_{t, z}^{(n-1)}(\gamma)\right)\right] \mathrm{d} z \mid \\
\leq & \max _{y \in \Re} \max _{0 \leq s \leq \tau}\left|\frac{1}{2 a} \int_{0}^{s} \mathrm{~d} t \int_{y-a(s-t)}^{y+a(s-t)}\left[g\left(t, z, X_{t, z}^{(n)}(\gamma)\right)-g\left(t, z, X_{t, z}^{(n-1)}(\gamma)\right)\right] \mathrm{d} z\right| \\
& +\max _{y \in \Re} \max _{0 \leq s \leq \tau}\left|\frac{1}{2 a} \int_{0}^{s} \mathrm{~d} C_{t}(\gamma) \int_{y-a(s-t)}^{y+a(s-t)}\left[h\left(t, z, X_{t, z}^{(n)}(\gamma)\right)-h\left(t, z, X_{t, z}^{(n-1)}(\gamma)\right)\right] \mathrm{d} z\right| \\
\leq & \max _{y \in \Re} \max _{0 \leq s \leq \tau} \frac{1}{2 a} \int_{0}^{s} \mathrm{~d} t \int_{y-a(s-t)}^{y+a(s-t)}\left|g\left(t, z, X_{t, z}^{(n)}(\gamma)\right)-g\left(t, z, X_{t, z}^{(n-1)}(\gamma)\right)\right| \mathrm{d} z \\
& +\max _{y \in \Re} \max _{0 \leq s \leq \tau} \frac{1}{2 a} \int_{0}^{s} \mathrm{~d} C_{t}(\gamma) \int_{y-a(s-t)}^{y+a(s-t)}\left|h\left(t, z, X_{t, z}^{(n)}(\gamma)\right)-h\left(t, z, X_{t, z}^{(n-1)}(\gamma)\right)\right| \mathrm{d} z \\
\leq & \max _{y \in \Re} \max _{0 \leq s \leq \tau} \frac{1}{2 a} \int_{0}^{s} \mathrm{~d} t \int_{y-a(s-t)}^{y+a(s-t)}\left|g\left(t, z, X_{t, z}^{(n)}(\gamma)\right)-g\left(t, z, X_{t, z}^{(n-1)}(\gamma)\right)\right| \mathrm{d} z \\
& +\max _{y \in \Re} \max _{0 \leq s \leq \tau} \frac{1}{2 a} \int_{0}^{s} \mathrm{~d} t K_{\gamma} \int_{y-a(s-t)}^{y+a(s-t)}\left|h\left(t, z, X_{t, z}^{(n)}(\gamma)\right)-h\left(t, z, X_{t, z}^{(n-1)}(\gamma)\right)\right| \mathrm{d} z .
\end{aligned}
$$

According to Equation (14) for $k=n-1$ and Equation (8), we obtain

$$
\begin{aligned}
D_{\tau}^{(n)}(\gamma) \leq & \left.\left.\max _{y \in \Re} \max _{0 \leq s \leq \tau} \frac{1}{2 a} \int_{0}^{s} \mathrm{~d} t \int_{y-a(s-t)}^{y+a(s-t)} L \mid X_{t, z}^{(n)}(\gamma)\right)-X_{t, z}^{(n-1)}(\gamma)\right) \mid \mathrm{d} z \\
& \left.\left.+\max _{y \in \Re} \max _{0 \leq s \leq \tau} \frac{K_{\gamma}}{2 a} \int_{0}^{s} \mathrm{~d} t \int_{y-a(s-t)}^{y+a(s-t)} L \mid X_{t, z}^{(n)}(\gamma)\right)-X_{t, z}^{(n-1)}(\gamma)\right) \mid \mathrm{d} z \\
= & \left.\left.\frac{1+K_{\gamma}}{2 a} \max _{y \in \Re} \max _{0 \leq s \leq \tau} \int_{0}^{s} \mathrm{~d} t \int_{y-a(s-t)}^{y+a(s-t)} \mid X_{t, z}^{(n)}(\gamma)\right)-X_{t, z}^{(n-1)}(\gamma)\right) \mid \mathrm{d} z \\
\leq & \frac{1+K_{\gamma}}{2 a} L \max _{y \in \Re} \max _{0 \leq s \leq \tau} \int_{0}^{s} \mathrm{~d} t \int_{y-a(s-t)}^{y+a(s-t)}\left[\frac{2 L^{n-1}\left(1+K_{\gamma}\right)^{n-1} N}{(2 n-2) !} \tau^{2 n-2}\right. \\
& \left.+\frac{L^{n-1}\left(1+K_{\gamma}\right)^{n-1} Q}{(2 n-1) !} \tau^{2 n-1}+\frac{L^{n}\left(1+K_{\gamma}\right)^{n}(1+P)}{(2 n) !} \tau^{2 n}\right] \mathrm{d} z \\
\leq & \frac{1+K_{\gamma}}{2 a} L \int_{0}^{\tau} 2 a(\tau-t)\left[\frac{2 L^{n-1}\left(1+K_{\gamma}\right)^{n-1} P}{(2 n-2) !} \tau^{2 n-2}+\frac{L^{n-1}\left(1+K_{\gamma}\right)^{n-1} Q}{(2 n-1) !} \tau^{2 n-1}\right. \\
& \left.+\frac{L^{n}\left(1+K_{\gamma}\right)^{n}(1+P)}{(2 n) !} \tau^{2 n}\right] \mathrm{d} t \\
= & \frac{2 L^{n}\left(1+K_{\gamma}\right)^{n} P}{(2 n) !} \tau^{2 n}+\frac{L^{n}\left(1+K_{\gamma}\right)^{n} Q}{(2 n+1) !} \tau^{2 n+1}+\frac{L^{n+1}\left(1+K_{\gamma}\right)^{n+1}(1+P)}{(2 n+2) !} \tau^{2 n+2} .
\end{aligned}
$$

Thus, we show that Equation (14) is true for all $n$. For any sample $\gamma$, noting that

$$
\begin{aligned}
\sum_{n=0}^{\infty} D_{\tau}^{(n)}(\gamma) \leq \sum_{n=0}^{\infty} & \frac{2 L^{n}\left(1+K_{\gamma}\right)^{n} P}{(2 n) !} T^{2 n}+\frac{L^{n}\left(1+K_{\gamma}\right)^{n} Q}{(2 n+1) !} T^{2 n+1} \\
& +\frac{L^{n+1}\left(1+K_{\gamma}\right)^{n+1}(1+P)}{(2 n+2) !} T^{2 n+2}
\end{aligned}
$$


Clearly, according to Weierstrass' criterion, we know that $X_{\tau, y}^{(n)}(\gamma)$ uniformly converges on $[0, T]$ for every given $\gamma$.

Denote

$$
X_{\tau, y}(\gamma)=\lim _{n \rightarrow \infty} X_{\tau, y}^{(n)}(\gamma), \gamma \in \Gamma, \tau \in[0, T]
$$

Then, we have

$$
\begin{aligned}
X_{\tau, y}(\gamma)=\frac{1}{2}[ & \psi(y+a \tau)+\psi(y-a \tau)]+\frac{1}{2 a} \int_{y-a \tau}^{y+a \tau} \varphi(z) \mathrm{d} z+\frac{1}{2 a} \int_{0}^{\tau} \mathrm{d} t \int_{y-a(\tau-t)}^{y+a(\tau-t)} g\left(t, z, X_{t, z}\right) \mathrm{d} z \\
& +\frac{1}{2 a} \int_{0}^{\tau} \mathrm{d} C_{t}(\gamma) \int_{y-a(\tau-t)}^{y+a(\tau-t)} h\left(t, z, X_{t, z}\right) \mathrm{d} z .
\end{aligned}
$$

Hence, for all $\tau>0$, Equation (5) has the solution $X_{\tau, y}$ because of $T$ being arbitrary.

Next, uniqueness is going to be proved. Assume that the uncertain equation has two solutions $X_{\tau, y}$ and $X_{\tau, y}^{*}$. According to Equations (9) and (8), we have

$$
\begin{aligned}
& \left|X_{\tau, y}(\gamma)-X_{\tau, y}^{*}(\gamma)\right| \\
& =\mid \frac{1}{2 a} \int_{0}^{\tau} \mathrm{d} t \int_{y-a(\tau-t)}^{y+a(\tau-t)}\left[g\left(t, z, X_{t, z}\right)-g\left(t, z, X_{t, z}^{*}\right)\right] \mathrm{d} z \\
& \quad+\frac{1}{2 a} \int_{0}^{\tau} \mathrm{d} C_{\tau}(\gamma) \int_{y-a(\tau-t)}^{y+a(\tau-t)}\left[h\left(t, z, X_{t, z}\right)-h\left(t, z, X_{t, z}^{*}\right)\right] \mathrm{d} z \mid \\
& \leq \frac{1}{2 a} \int_{0}^{\tau} \mathrm{d} t \int_{y-a(\tau-t)}^{y+a(\tau-t)}\left|g\left(t, z, X_{t, z}\right)-g\left(t, z, X_{t, z}^{*}\right)\right| \mathrm{d} z \\
& \quad+\frac{1}{2 a} \int_{0}^{\tau} \mathrm{d} C_{\tau}(\gamma) \int_{y-a(\tau-t)}^{y+a(\tau-t)}\left|h\left(t, z, X_{t, z}\right)-h\left(t, z, X_{t, z}^{*}\right)\right| \mathrm{d} z \\
& \leq L\left(1+K_{\gamma}\right) \int_{0}^{\tau} \mathrm{d} t \int_{y-a(\tau-t)}^{y+a(\tau-t)}\left|X_{t, z}(\gamma)-X_{t, z}^{*}(\gamma)\right| \mathrm{d} z .
\end{aligned}
$$

It follows from the Grönwall inequality that

$$
\left|X_{\tau, y}(\gamma)-X_{\tau, y}^{*}(\gamma)\right| \leq 0
$$

Therefore, $X_{\tau, y}=X_{\tau, y}^{*}$. Thus, we prove the uniqueness.

Finally, we will verify that $X_{\tau, y}$ is sample-continuous. According to the above proof,

$$
\begin{aligned}
& X_{\tau, y}(\gamma) \\
& \leq \sum_{n=0}^{\infty}\left[\frac{2 L^{n}\left(1+K_{\gamma}\right)^{n} P}{(2 n) !} \tau^{2 n}+\frac{L^{n}\left(1+K_{\gamma}\right)^{n} Q}{(2 n+1) !} \tau^{2 n+1}+\frac{L^{n+1}\left(1+K_{\gamma}\right)^{n+1}(1+P)}{(2 n+2) !} \tau^{2 n+2}\right] \\
& \leq(1+2 P+Q) \exp \left(\sqrt{L\left(1+K_{\gamma}\right) \tau}\right)
\end{aligned}
$$

holds. Suppose that $0<s<\tau$ and $z<y$, we have 


$$
\begin{aligned}
& \left|X_{\tau, y}(\gamma)-X_{s, z}^{*}(\gamma)\right| \\
& =\mid \frac{1}{2 a} \int_{0}^{\tau} \mathrm{d} t \int_{y-a(\tau-t)}^{y+a(\tau-t)} g\left(t, v, X_{t, v}\right) \mathrm{d} v-\frac{1}{2 a} \int_{0}^{s} \mathrm{~d} t \int_{z-a(s-\tau)}^{z+a(s-\tau)} g\left(t, v, X_{t, v}\right) \mathrm{d} v \\
& \quad+\frac{1}{2 a} \int_{0}^{\tau} \mathrm{d} C_{t}(\gamma) \int_{y-a(\tau-t)}^{y+a(\tau-t)} h\left(t, v, X_{t, v}\right) \mathrm{d} v-\frac{1}{2 a} \int_{0}^{s} \mathrm{~d} C_{t}(\gamma) \int_{z-a(s-t)}^{z+a(s-t)} h\left(t, v, X_{t, v}\right) \mathrm{d} v \mid \\
& =\frac{1}{2 a} \mid \int_{s}^{\tau} \mathrm{d} t \int_{y-a(\tau-t)}^{y+a(\tau-t)} g\left(t, v, X_{t, v}\right) \mathrm{d} v-\int_{0}^{s} \mathrm{~d} t \int_{z-a(s-t)}^{y-a(\tau-t)} g\left(t, v, X_{t, v}\right) \mathrm{d} v \\
& \quad+\int_{0}^{s} \mathrm{~d} t \int_{z+a(s-t)}^{y+a(\tau-t)} g\left(t, v, X_{t, v}\right) \mathrm{d} v+\int_{s}^{t} \mathrm{~d} C_{t}(\gamma) \int_{y-a(\tau-t)}^{y+a(\tau-t)} h\left(t, v, X_{t, v}\right) \mathrm{d} v \\
& \quad-\int_{0}^{s} \mathrm{~d} C_{t}(\gamma) \int_{z-a(s-t)}^{y-a(\tau-t)} h\left(t, v, X_{t, v}\right) \mathrm{d} v+\int_{0}^{s} \mathrm{~d} C_{t}(\gamma) \int_{z+a(s-t)}^{y+a(\tau-t)} h\left(t, v, X_{t, v}\right) \mathrm{d} v \mid
\end{aligned}
$$

according to the interval additivity of the integral. By using Lemma 1, Equations (9) and (24), we have

$$
\begin{aligned}
& \left|X_{\tau, y}(\gamma)-X_{s, z}^{*}(\gamma)\right| \\
& \leq \frac{1}{2 a}\left[\int_{s}^{\tau} \mathrm{d} t \int_{y-a(\tau-t)}^{y+a(\tau-t)}\left|g\left(t, v, X_{t, v}\right)\right| \mathrm{d} v+\int_{0}^{s} \mathrm{~d} t \int_{z-a(s-t)}^{y-a(\tau-t)}\left|g\left(t, v, X_{t, v}\right)\right| \mathrm{d} v\right. \\
& \quad+\int_{0}^{s} \mathrm{~d} t \int_{z+a(s-t)}^{y+a(\tau-t)}\left|g\left(t, v, X_{t, v}\right)\right| \mathrm{d} v+\int_{s}^{\tau} \mathrm{d} t K_{\gamma} \int_{y-a(\tau-t)}^{y+a(\tau-t)}\left|h\left(t, v, X_{t, v}\right)\right| \mathrm{d} v \\
& \left.\quad+\int_{0}^{s} \mathrm{~d} t K_{\gamma} \int_{z-a(s-t)}^{y-a(\tau-t)}\left|h\left(t, v, X_{t, v}\right)\right| \mathrm{d} v+\int_{0}^{s} \mathrm{~d} t K_{\gamma} \int_{z+a(s-t)}^{y+a(\tau-t)}\left|h\left(t, v, X_{t, v}\right)\right| \mathrm{d} v\right] \\
& \leq \frac{1}{2 a}\left(1+K_{\gamma}\right) L\left[\int_{s}^{\tau} \mathrm{d} t \int_{y-a(\tau-t)}^{y+a(\tau-t)}\left(1+\left|X_{t, v}\right|\right) \mathrm{d} v+\int_{0}^{s} \mathrm{~d} t \int_{z-a(s-t)}^{y-a(\tau-t)}\left(1+\left|X_{t, v}\right|\right) \mathrm{d} v\right. \\
& \left.\quad+\int_{0}^{s} \mathrm{~d} t \int_{z+a(s-t)}^{y+a(\tau-t)}\left(1+\left|X_{t, v}\right|\right) \mathrm{d} v\right] \\
& \leq \frac{(1+2 P+Q) \exp \left(\sqrt{L\left(1+K_{\gamma}\right)} \tau\right)}{2}\left[(\tau-s)^{2}+\frac{1}{a} s[(y-z)-a(\tau-s)]\right. \\
& \left.\quad+\frac{1}{a} s[(y-z)+a(\tau-s)]\right] \\
& =\frac{(1+2 P+Q) \exp \left(\sqrt{L\left(1+K_{\gamma}\right)} \tau\right)}{2}\left[(\tau-s)^{2}+\frac{2}{a} s(y-z)\right] .
\end{aligned}
$$

Thus $\left|X_{\tau, y}-X_{s, z}^{*}\right| \rightarrow 0$ as $(s, z) \rightarrow(\tau, y)$. That is, $X_{\tau, y}$ is sample-continuous. Thus, we proved the theorem.

Example 1. Considering

$$
\frac{\partial^{2} X}{\partial \tau^{2}}(\tau, y)=a^{2} \frac{\partial^{2} X}{\partial y^{2}}(\tau, y)+X(\tau, y)+\dot{C_{\tau}}, X(0, y)=0, \frac{\partial X}{\partial \tau}(0, y)=0,
$$

Then, the unique solution of Equation (27) is

$$
X(\tau, y)=\frac{1}{2 a} \int_{0}^{\tau} \mathrm{d} t \int_{y-a(\tau-t)}^{y+a(\tau-t)} X(t, z) \mathrm{d} z+\int_{0}^{\tau}(\tau-t) \mathrm{d} C_{t}
$$

according to Theorem 1. 
Proof. Now we verify that Equation (28) is the solution of Equation (27). Obviously, we have $X(0, y)=0$ from Equation (28). Noting that

$$
\begin{aligned}
\frac{\partial X}{\partial \tau}(\tau, y) & =\frac{1}{2 a}\left[\frac{\partial}{\partial \tau} \int_{0}^{\tau} \mathrm{d} C_{t} \int_{y-a(\tau-t)}^{y+a(\tau-t)} X(t, z) \mathrm{d} z\right] \\
& =\frac{1}{2} \int_{0}^{\tau}[X(t, y+a(\tau-t))+X(t, y-a(\tau-t))] \mathrm{d} t+C_{\tau}
\end{aligned}
$$

we have

$$
\frac{\partial X}{\partial \tau}(0, y)=0
$$

Since

$$
\begin{aligned}
\frac{\partial^{2} X}{\partial \tau^{2}}(\tau, y) & =\frac{\partial}{\partial \tau}\left[\frac{1}{2} \int_{0}^{\tau}[X(t, y+a(\tau-t))+X(t, y-a(\tau-t))] \mathrm{d} t+C_{\tau}\right] \\
& =X(\tau, y)+\frac{a}{2} \int_{0}^{\tau}\left[\frac{\partial X}{\partial y}(t, y+a(\tau-t))-\frac{\partial X}{\partial y}(t, y-a(\tau-t))\right] \mathrm{d} t+\frac{\mathrm{d} C_{\tau}}{\mathrm{d} \tau} \\
\frac{\partial X}{\partial y}(\tau, y) & =\frac{\partial}{\partial x}\left[\frac{1}{2 a} \int_{0}^{\tau} \mathrm{d} t \int_{y-a(\tau-t)}^{y+a(\tau-t)} X(t, z) \mathrm{d} z+\int_{0}^{\tau}(\tau-t) \mathrm{d} C_{t}\right] \\
& =\frac{1}{2 a} \int_{0}^{\tau}[X(t, y+a(\tau-t))-X(t, y-a(\tau-t))] \mathrm{d} t
\end{aligned}
$$

and

$$
\begin{aligned}
\frac{\partial^{2} X}{\partial y^{2}}(\tau, y) & =\frac{1}{2 a}\left[\frac{\partial}{\partial y} \int_{0}^{\tau}[X(t, y+a(\tau-t))-X(t, y-a(\tau-t))] \mathrm{d} t\right] \\
& =\frac{1}{2 a} \int_{0}^{\tau}\left[\frac{\partial X}{\partial y}(t, y+a(\tau-t))-\frac{\partial X}{\partial y}(t, y-a(\tau-t))\right] \mathrm{d} t .
\end{aligned}
$$

Then, we have

$$
\frac{\partial^{2} X}{\partial \tau^{2}}(\tau, y)=a^{2} \frac{\partial^{2} X}{\partial y^{2}}(\tau, y)+X(\tau, y)+\dot{C_{\tau}}
$$

Thus, we prove that Equation (28) is the solution of Equation (27). Next we prove the uniqueness. Since $g(\tau, y, x)=x$ and $h(\tau, y, x)=1$, we obtain

$$
|g(\tau, y, x)|+|h(\tau, y, x)|=1+|x|, \quad \forall x \in \Re,
$$

and

$$
|g(\tau, y, x)-g(\tau, y, v)|+|h(\tau, y, x)-h(\tau, y, v)|=|x-v|, \quad \forall x, v \in \Re .
$$

Due to Theorem 1, Equation (27) has a unique solution which is Equation (28).

Example 2. The solution of the following uncertain wave equation

$$
\frac{\partial^{2} X}{\partial \tau^{2}}(\tau, y)=a^{2} \frac{\partial^{2} X}{\partial y^{2}}(\tau, y)+\dot{C_{\tau}}, X(0, y)=\sin y, \frac{\partial X}{\partial \tau}(0, y)=0 .
$$

is

$$
X(\tau, y)=\sin y \cos a \tau+\int_{0}^{\tau}(\tau-t) \mathrm{d} C_{t}
$$

and it is unique from Theorem 1 .

Proof. Obviously, we have $X(0, y)=\sin y$ from Equation (38). Noting that

$$
\frac{\partial X}{\partial \tau}(\tau, y)=\frac{\partial}{\partial \tau}\left[\sin y \cos a \tau+\int_{0}^{\tau}(\tau-t) \mathrm{d} C_{t}\right]=-a \sin y y \sin a \tau+C_{\tau},
$$


we have

$$
\frac{\partial X}{\partial \tau}(0, y)=0
$$

Since

$$
\begin{gathered}
\frac{\partial^{2} X}{\partial \tau^{2}}(\tau, y)=\frac{\partial}{\partial \tau}\left[-a \sin y \sin a \tau+C_{\tau}\right]=-a^{2} \sin y \cos a \tau+\frac{\mathrm{d} C_{\tau}}{\mathrm{d} \tau}, \\
\frac{\partial X}{\partial y}(\tau, y)=\frac{\partial}{\partial y}\left[\sin y \cos a \tau+\int_{0}^{\tau}(\tau-t) \mathrm{d} C_{t}\right]=\cos y \cos a \tau+\int_{0}^{\tau}(\tau-t) \mathrm{d} C_{t},
\end{gathered}
$$

and

$$
\frac{\partial^{2} X}{\partial y^{2}}(\tau, y)=\frac{\partial}{\partial y}\left[\cos y \cos a \tau+\int_{0}^{\tau}(\tau-t) \mathrm{d} C_{t}\right]=\sin y \cos a \tau+\int_{0}^{\tau}(\tau-t) \mathrm{d} C_{t}
$$

we have

$$
\frac{\partial^{2} X}{\partial \tau^{2}}(\tau, y)=a^{2} \frac{\partial^{2} X}{\partial y^{2}}(\tau, y)+\dot{C_{\tau}}
$$

Thus, we prove that Equation (38) is the solution of Equation (37). Next, we prove the uniqueness. Since $g(\tau, y, x)=0$ and $h(\tau, y, x)=1$, we obtain

$$
|g(\tau, y, x)|+|h(\tau, y, x)|=1 \leq(1+|x|), \quad \forall x \in \Re,
$$

and

$$
|g(\tau, y, x)-g(\tau, y, v)|+|h(\tau, y, x)-h(\tau, y, v)|=0, \forall x, v \in \Re .
$$

According to Theorem 1, Equation (37) has a unique solution which is Equation (38).

Example 3. Considering

$$
\frac{\partial^{2} X}{\partial \tau^{2}}(\tau, y)=a^{2} \frac{\partial^{2} X}{\partial y^{2}}(\tau, y)+\sin ^{2} X(\tau, y)+\dot{C}_{\tau}, X(0, y)=0, \quad \frac{\partial X}{\partial \tau}(0, y)=0,
$$

Then, the unique solution of Equation (47) is

$$
X(\tau, y)=\frac{1}{2 a} \int_{0}^{\tau} \mathrm{d} t \int_{y-a(\tau-t)}^{y+a(\tau-t)} \sin ^{2} X(t, z) \mathrm{d} z+\int_{0}^{\tau}(\tau-t) \mathrm{d} C_{t}
$$

according to Theorem 1.

Proof. Now we verify that Equation (48) is the solution of Equation (47). Obviously, we have $X(0, y)=0$ from Equation (48). Noting that

$$
\begin{aligned}
\frac{\partial X}{\partial \tau}(\tau, y)= & \frac{1}{2 a}\left[\frac{\partial}{\partial \tau} \int_{0}^{\tau} \mathrm{d} C_{t} \int_{y-a(\tau-t)}^{y+a(\tau-t)} \sin ^{2} X(t, z) \mathrm{d} z\right] \\
& =\frac{1}{2} \int_{0}^{\tau}\left[\sin ^{2} X(t, y+a(\tau-t))+X(t, y-a(\tau-t))\right] \mathrm{d} t+C_{\tau},
\end{aligned}
$$

we have

$$
\frac{\partial X}{\partial \tau}(0, y)=0
$$

Since 


$$
\begin{aligned}
& \frac{\partial^{2} X}{\partial \tau^{2}}(\tau, y) \\
&=\frac{\partial}{\partial \tau}\left[\frac{1}{2} \int_{0}^{\tau}\left[\sin ^{2} X(t, y+a(\tau-t))+\sin ^{2} X(t, y-a(\tau-t))\right] \mathrm{d} t+C_{\tau}\right] \\
&=\sin ^{2} X(\tau, y)+a \int_{0}^{\tau}\left[\sin X(t, y+a(\tau-t)) \cos X(t, y+a(\tau-t)) \frac{\partial X}{\partial y}(t, y+a(\tau-t))\right. \\
&\left.-\sin X(t, y-a(\tau-t)) \cos X(t, y-a(\tau-t)) \frac{\partial X}{\partial y}(t, y-a(\tau-t))\right] \mathrm{d} t+\frac{\mathrm{d} C_{\tau}}{\mathrm{d} \tau}, \\
& \frac{\partial X}{\partial y}(\tau, y)=\frac{\partial}{\partial x}\left[\frac{1}{2 a} \int_{0}^{\tau} \mathrm{d} t \int_{y-a(\tau-t)}^{y+a(\tau-t)} \sin ^{2} X(t, z) \mathrm{d} z+\int_{0}^{\tau}(\tau-t) \mathrm{d} C_{t}\right] \\
&=\frac{1}{2 a} \int_{0}^{\tau}\left[\sin ^{2} X(t, y+a(\tau-t))-\sin ^{2} X(t, y-a(\tau-t))\right] \mathrm{d} t,
\end{aligned}
$$

and

$$
\begin{aligned}
& \frac{\partial^{2} X}{\partial y^{2}}(\tau, y) \\
& =\frac{1}{2 a}\left[\frac{\partial}{\partial y} \int_{0}^{\tau}\left[\sin ^{2} X(t, y+a(\tau-t))-\sin ^{2} X(t, y-a(\tau-t))\right] \mathrm{d} t\right] \\
& =\frac{1}{a} \int_{0}^{\tau}\left[\sin X(t, y+a(\tau-t)) \cos X(t, y+a(\tau-t)) \frac{\partial X}{\partial y}(t, y+a(\tau-t))\right. \\
& \left.\quad-\sin X(t, y-a(\tau-t)) \cos X(t, y-a(\tau-t)) \frac{\partial X}{\partial y}(t, y-a(\tau-t))\right] \mathrm{d} t .
\end{aligned}
$$

Then, we have

$$
\frac{\partial^{2} X}{\partial \tau^{2}}(\tau, y)=a^{2} \frac{\partial^{2} X}{\partial y^{2}}(\tau, y)+\sin ^{2} X(\tau, y)+\dot{C_{\tau}} .
$$

Thus, we prove that Equation (48) is the solution of Equation (47). Next we prove the uniqueness. Since $g(\tau, y, x)=\sin ^{2} x$ and $h(\tau, y, x)=1$, we obtain

$$
|g(\tau, y, x)|+|h(\tau, y, x)|=1+\sin ^{2} x \leq(1+|x|), \forall x \in \Re,
$$

and

$$
|g(\tau, y, x)-g(\tau, y, v)|+|h(\tau, y, x)-h(\tau, y, v)| \leq 2|x-v|, \quad \forall x, v \in \Re .
$$

Due to Theorem 1, Equation (47) has a unique solution which is Equation (48).

Corollary 1. The solution of Equation (5) is unique if functions $\psi(x)$ and $\varphi(x)$ satisfy

$$
|\psi(y)| \leq P,|\varphi(y)| \leq Q, \forall y \in \Re
$$

for $P, Q>0$, and $g(\tau, y, x)$ and $h(\tau, y, x)$ are bounded and its partial differential is continuous in relation to $x$. Furthermore, the solution of Equation (5) is sample-continuous.

Proof. There is a crisp number $K$ such that

$$
|g(\tau, y, x)| \leq K, \text { and }|h(\tau, y, x)| \leq K, \forall x \in \Re,
$$

due to $g(\tau, y, x)$ and $h(\tau, y, x)$ being bounded in relation to $x$. Thus we have

$$
|g(\tau, y, x)|+|h(\tau, y, x)| \leq 2 K \leq 2 K(1+|x|) .
$$


Since the partial differential of $g(\tau, y, x)$ is continuous with in relation to $x$, there is a crisp number $\mathrm{H}$ such that

$$
\left|\frac{\partial g}{\partial X}(\tau, y, x)\right| \leq H, \quad \forall x \in \Re .
$$

It follows from the Lagrange Mean Value Theorem that

$$
|g(\tau, y, x)-g(\tau, y, v)|=\left|\frac{\partial g(\tau, y, x)}{\partial x}(x-v)\right| \leq H|x-v|, \quad \forall x, v \in \Re .
$$

Denoting $L=\max \{2 K, H\}$, we derive the result via Theorem 1 . In addition the proof of sample-continuity for the solution is as the same as one in Theorem 1 . Therefore the proof is completed.

Corollary 2. The solution of the following linear uncertain wave equation

$$
\frac{\partial^{2} X}{\partial \tau^{2}}(\tau, y)=a^{2} \frac{\partial^{2} X}{\partial y^{2}}(\tau, y)+g(\tau, y)+h(\tau, y) \dot{C_{\tau}}, \quad X(0, y)=\psi(y), \quad \frac{\partial X}{\partial \tau}(0, y)=\varphi(y)
$$

is unique if functions $\psi(y)$ and $\varphi(y)$ are bounded

$$
|\psi(y)| \leq P, \quad|\varphi(x)| \leq Q, \forall y \in \Re
$$

for $P, Q>0$, and functions $g(\tau, y)$ and $h(\tau, y)$ are also bounded. Furthermore, the solution of Equation (62) is sample-continuous.

Proof. There is a crisp number $K$ such that

$$
|g(\tau, y, x)| \leq K, \text { and }|h(\tau, y, x)| \leq K, \forall x \in \Re,
$$

because $g(\tau, y)$ and $h(\tau, y)$ are bounded. Following that, we have

$$
|g(\tau, y, x)|+|h(\tau, y, x)| \leq 2 K \leq 2 K(1+|x|) \stackrel{\text { Let } L=2 K}{=} L(1+|x|), \quad \forall x \in \Re .
$$

Denoting

$$
g(\tau, y, x)=g(\tau, y), \quad h(\tau, y, x)=h(\tau, y), \quad \forall x \in \Re,
$$

we have

$$
|g(\tau, y, x)-g(\tau, y, v)|+|h(\tau, y, x)-h(\tau, y, v)|=0, \quad \forall x, v \in \Re .
$$

Hence we obtain the corollary by using Theorem 1.

Remark 1. The proofs show that Corollarys 1 and 2 are special cases of Theorem 1 . Theorem 1 is more general and it is convenient for the complex case. However, Corollarys 1 and 2 are more convenient for special cases.

\section{Discussion and Conclusions}

The uncertain wave equation is established to model wave propagation with uncertain disturbances. For an equation, the property existence and uniqueness of the solution is important. Hence, the existence and uniqueness theorem was proposed and proved under the Lipschitz condition and linear growth condition. Then, some examples were given to illustrate how to obtain an uncertain wave equation's solution. Finally, two corollaries were derived, which is the special case of the general existence and uniqueness theorem and is convenient for judging whether the special wave equation has a unique solution or not.

Funding: This work was supported by National Natural Science Foundation of China (Grant No. 72101080), Natural Science Foundation of Hebei Province (Grant No. F2020202056), and the Key Project of Hebei Education Department (Grant No. ZD2020125). 
Institutional Review Board Statement: Not applicable.

Informed Consent Statement: Not applicable.

Data Availability Statement: Not Applicable.

Conflicts of Interest: The author declares that they have no conflict of interest.

\section{References}

1. Liu, B. Uncertainty Theory, 2nd ed.; Springer: Berlin, Germany, 2007.

2. Liu, B. Theory and Practice of Uncertain Programming, 2nd ed.; Springer: Berlin, Germany, 2009.

3. Gao, R.; Zhang, Z.M. Analysis of green supply chain considering green degree and sales effort with uncertain demand. J. Intell. Syst. 2020, 38, 4247-4264. [CrossRef]

4. Gao, R.; Yao, K. Importance index of components in uncertain random systems. Knowl.-Based Syst. 2016, 109, 208-217. [CrossRef]

5. Ahmadzade, H.; Gao, R. Covariance of uncertain random variables and its application to portfolio optimization. J. Ambient. Intell. Humaniz. Comput. 2020, 11, 2613-2624. [CrossRef]

6. Chen, L.; Gao, R.; Li, Z.Y.; Bian, Y.X. Elliptic entropy of uncertain random variables with application to portfolio selection. Soft Comput. 2021, 25, 1925-1939. [CrossRef]

7. Ning, Y.F.; Su, T.Y. A multilevel approach for modelling vehicle routing problem with uncertain travelling time. J. Intell. Manuf. 2017, 28, 683-688. [CrossRef]

8. Liu, Y.H.; Ralescu, D.A. Expected loss of uncertain random systems. Soft Comput. 2018, 22, 5573-5578. [CrossRef]

9. Liu, B. Fuzzy process, hybrid process and uncertain process. J. Uncertain Syst. 2008, 2, 3-16.

10. Liu, B. Some research problems in uncertainty theory. J. Uncertain Syst. 2009, 3, 3-10.

11. Chen, X.W.; Liu, B. Existence and uniqueness theorem for uncertain differential equations. Fuzzy Optim. Decis. Mak. 2010, 9, 69-81. [CrossRef]

12. Sheng, Y.H.; Wang, C.G. Stability in the $p$-th moment for uncertain differential equation. J. Intell. Fuzzy Syst. 2014, $26,1263-1271$. [CrossRef]

13. Yao, K.; Ke, H.; Sheng, Y.H. Stability in mean for uncertain differential equation. Fuzzy Optim. Decis. Mak. 2015, 14, 365-379. [CrossRef]

14. Yao, K.; Chen, X.W. A numerical method for solving uncertain differential equations. J. Intell. Fuzzy Syst. 2013, 25, 825-832. [CrossRef]

15. Gao, R. Milne method for solving uncertain differential equations. Appl. Math. Comput. 2016, 74, 774-785. [CrossRef]

16. Gao, R.; Wu, W.; Lang, C.; Lang, L.Y. Geometric Asian barrier option pricing formulas of uncertain stock model. Chaos Solitons Fractals 2020, 140, 110178. [CrossRef]

17. Zhu, Y.G. Uncertain optimal control with application to a portfolio selection model. Cybern. Syst. 2010, 41, 535-547. [CrossRef]

18. Walsh, J.B. An introduction to stochastic partial differential equations. In École d'Été de Probabilités de Saint Flour XIV. Lecture Notes in Mathematics; Springer: Berlin/Heidelberg, Germany, 1984; Volume 1180, pp. 265-439.

19. Cabaña, E.M. The vibrating string forced by white noise. Z. Wahrscheinlichkeitsthrorie Verwandte Geb. 1970, 15, 111-130. [CrossRef]

20. Orsingher, E. Randomly forced vibrations of a string. Annales de l'Institut Henri Poincaré-Sect. B 1982, 18, 367-394.

21. Marcus, M.; Mizel, V.J. Stochastic hyperbolic systems and the wave equation. Stoch. Stoch. Rep. 1991, 36, 225-244. [CrossRef]

22. Czapor, S.R.; Mclenaghan, R.G.; Sasse, F.D. Complete solution of Hadamard's problem for the scalar wave equation on Petrov type III spacetimes. Ann. l'Institut Henri Poincaré 1999, 71, 595-620.

23. Yang, X.F.; Yao, K. Uncertain partial differential equation with application to heat conduction. Fuzzy Optim. Decis. Mak. 2017, 16, 379-403. [CrossRef]

24. Gao, R.; Ralescu, A.D. Uncertain wave equation for string vibration. Trans. Fuzzy Syst. 2019, 27, 1323-1331. [CrossRef]

25. Liu, B. Uncertain distribution and independence of uncertain processes. Fuzzy Optim. Decis. Mak. 2014, 13, 259-271. [CrossRef] 